\title{
Microorganisms and biosorption of heavy metals in the environment: a review paper
}

\begin{abstract}
Industrial waste water and sediment containing heavy metals causes many ecological and health related problems. Many conventional methods were already being used to decontaminate the environment from adverse effect of these pollutants but yet most of the methods used are very expensive and far away from their best possible performance. The capability of microorganisms to bind metal ions is a well-known trend. Different experimental data are documented and presented for different metals and biomass types. In this review a brief overview of the potential of biosorbents and biosorption processes were critically reviewed. This briefly describes biosorption process and some of the analysis of different lowcost biosorbents used for heavy metal remediation from waste stream.
\end{abstract}

Keyword: Microbes; Potential; Heavy metals; Biosorption 